\title{
Cyclic loading of a cellulose/hydrogel composite increases its fracture strength
}

\author{
Céline Samira Wyss ${ }^{\mathrm{a}, \mathrm{b}}$, Peyman Karami ${ }^{\mathrm{b}}$, Pierre-Etienne Bourban ${ }^{\mathrm{a}, *}$, Dominique \\ P. Pioletti ${ }^{\mathrm{b}}$ \\ a Laboratory for Processing of Advanced Composites, EPFL, Station 12, CH-1015, Lausanne, Switzerland \\ ${ }^{\mathrm{b}}$ Laboratory of Biomechanical Orthopedics, EPFL, Station 9, CH-1015, Lausanne, Switzerland
}

\section{GRAPHICAL ABSTRACT}

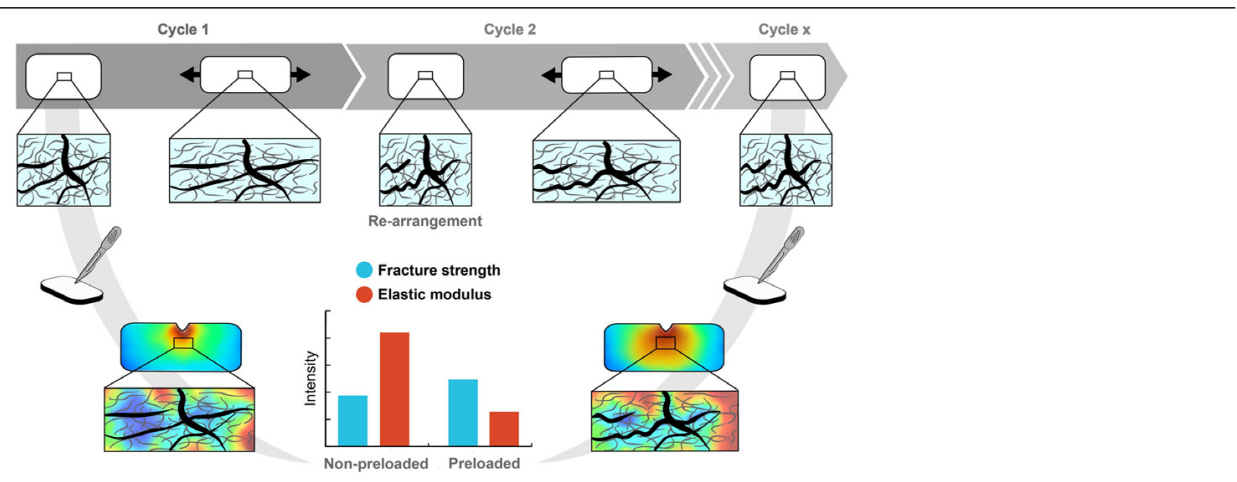

\section{A R T I C L E I N F O}

\section{Article history:}

Received 13 July 2018

Received in revised form 12 September 2018

Accepted 19 September 2018

Available online $\mathrm{xxxx}$

\section{Keywords:}

Hydrogel composite

Nano-fibrillated cellulose fibers

Mullins effect

Fracture mechanics

Digital image correlation

Confocal microscopy

\begin{abstract}
A B S T R A C T
The fracture properties of hydrogels which present similar characteristics to the Mullins effect is expected to decrease under repeated cyclic loading. Therefore, we assessed how cyclic loading affects the fracture behavior, the distribution of strain fields and the microstructure of hydrogel composites reinforced with nano-fibrillated cellulose fibers. Surprisingly, we observed that preloading before the creation of a crack in the hydrogel composite increased the fracture strength of pre-notched samples, while the corresponding fracture energy decreased. To understand this behavior, a digital image correlation analysis at the macro- and microscopic scale was performed to obtain local information on the strain field. In addition, the morphology of cellulose fibers was directly observed through fluorescence confocal microscopy before and after cyclic loading at different maximal applied strains. Microscopy results show that cyclic loading re-arrange the fiber network and relax local residual stresses in the hydrogel composite. The re-arrangement of the fiber network decreases the overall elastic modulus and correspondingly the fracture energy. However, this phenomenon helps the hydrogel composite to accommodate larger strains before the crack starts to propagate, which subsequently improves the fracture strength of pre-notched samples.
\end{abstract}

(c) 2018 Elsevier Ltd. All rights reserved.

\section{Introduction}

Hydrogels are suitable biomaterials for a wide range of biomedical applications [1] such as drug delivery [2], tissue engineering [3,

* Corresponding author.

E-mail address: pierre-etienne.bourban@epfl.ch (P.-E. Bourban).
4] or tissue repair [5]. In particular, load-bearing hydrogels gain attention [6] because the patient could rapidly put weight on the implant after its implementation satisfying then what has been defined as functional tissue engineering [7]. Moreover, tailoring mechanical properties of hydrogels to the native tissues may positively influence the formation of new tissues [8-11]. 
Since the mechanical properties of conventional hydrogels are known to be relatively low [12], more complex hydrogel structures such as dual crosslink hydrogels $[13,14]$ double network $[15,16]$ or hydrogel composites $[17,18]$ are employed to significantly improve their stiffness and toughness.

Recently, poly(ethylene glycol) dimethacrylate (PEGDM) hydrogels reinforced with nano-fibrillated cellulose (NFC) fibers were developed as permanent load-bearing implants [19]. A composite approach was adopted allowing the hydrogel to be injected and cured in situ in one-pot. Moreover, physical and mechanical properties of the hydrogel could be tuned by varying the concentration of reinforcement [20]. The long-term reliability of the hydrogel composite was evaluated under high-cyclic fatigue loading. Specifically, 10 million compression cycles at $20 \%$ strain were applied [21]. As for other tough hydrogels based on double network [22,23], a softening behavior appeared after the first loading cycle [21,24], demonstrated by an initial decrease of $10 \%$ of the elastic modulus. Furthermore, low-cycle fatigue tests showed that the softening appeared for strain lower or equal to the maximum strain ever applied. This phenomenon is usually referred to the Mullins effect and is mostly related to some breaking of bonds or re-arrangement of the material microstructure [22,25,26]. Those changes in the material may affect also other properties such as the swelling ratio [21] or the toughness [27]. However only a few studies evaluated the evolution of different mechanical or physical properties of hydrogels under cyclic loading [28]. Recently, fatigue fracture were performed on simple [29], double network [30,31] and self-recovery hydrogels [32] with the conclusion that the fracture behavior of the tested hydrogels is sensitive to fatigue loading. In particular, the fracture energy of double network hydrogel progressively reduces after each loading cycles.

Since PEGDM-NFC hydrogel composite shows similar softening behavior to double network hydrogels, we expected that its fracture properties would be reduced as well after being cyclically loaded. To clarify this and to understand the governing phenomena behind the observed Mullins effect, we evaluated in this work the fracture properties of PEGDM-NFC hydrogel composite and investigated how cyclic loading affects the fracture strength and energy of pre-notched samples, the distribution of the strain fields and the microstructure of the hydrogel. For that purpose, a multiscale analysis was adopted as represented in Fig. 1. The swollen hydrogel composites were either cyclically preloaded or not before to perform fracture tests (Fig. 1a). The overall fracture properties of the hydrogels were then measured with a single edge notch test (Fig. 1e). In parallel, digital image correlation (DIC) analysis was performed at macro- and microscopic level (Fig. 1d) in order to obtain local information about the distribution of strain fields. Finally, the morphology of the NFC network in the hydrogel composites was observed after different preloading conditions with fluorescence confocal microscopy (Fig. 1b).

\section{Materials and methods}

\subsection{Materials}

20 kDa Poly(ethylene glycol) dimethacrylate (PEGDM) was purchased by Polysciences (ref. 25406-25, Germany). Nano-fibrillated cellulose (NFC) from bleached softwood pulp (Elemental Chlorine Free (ECF), Zellstoff Stendal, Germany) were prepared as reported in [20]. SEM analysis shows that the NFC measure a few micrometers with a diameters in the range of $2-100 \mathrm{~nm}$ [33].

\subsection{Hydrogel synthesis}

Hydrogels were synthesized according to an established protocol [20]. Distilled water was added to $10 \mathrm{wt} \%$ of PEGDM, 0.5 vol.\% of NFC fibers and $0.1 \mathrm{~g} \mathrm{ml}^{-1} \%$ of Irgacure 2959 (BASF). The precursor of hydrogels composite was then mixed with the Ultra Turrax (IKA T25 digital, SN 25 10G) at 12'000 RPS for $30 \mathrm{~min}$ and degassed at 20 mbar to remove air bubbles. The homogenized precursor was casted in Teflon molds and covered with microscope slides before to cure for 30 min under UV-light irradiation with an ultra violet lamp (AxonLab) at a wavelength of $365 \mathrm{~nm}$ and an intensity of $5 \mathrm{~mW} \mathrm{~cm}^{-2}$. The geometries of the molds are shown in the supplementary on Fig. S1. The molds for single edge notch test are rectangular with the size of $30 \mathrm{~mm} \times 15 \mathrm{~mm} \times 2 \mathrm{~mm}$ (length $\times$ width $\times$ depth), while the dog bone molds used for simple tensile test measure $11 \mathrm{~mm} \times 5 \mathrm{~mm} \times 2 \mathrm{~mm}$.

The neat hydrogel corresponds to $10 \mathrm{wt} \%$ PEGDM hydrogel without NFC. All samples were tested in swollen state. The swelling ratios calculated from Eq. (1) are $165 \pm 6 \%$ and $79 \pm 9 \%$ for neat hydrogels and hydrogel composites respectively.

Swelling ratio $(\%)=100 *\left(\mathrm{~V}_{\text {swollen }}-\mathrm{V}_{\text {as synthesised }}\right) / \mathrm{V}_{\text {as synthesised }}$

\subsection{Cyclic tensile loading}

Cyclic tensile loading was performed on swollen neat hydrogels and hydrogel composites with the tensile machine (5 kN Zwicky equipped with a $100 \mathrm{~N}$ load cell, Zwick Roell, Germany) at a constant strain rate of $0.023 \mathrm{1} / \mathrm{s}$. The strain applied on dog bone samples, which corresponds to the relative deformations of their straight parts, started at $30 \%$ and was progressively increased by $20 \%$ up to $110 \%$.

\subsection{Preloading of hydrogels}

Cyclic tensile loading was performed on swollen neat hydrogels and hydrogel composites with the tensile machine Zwicky at a constant strain rate of $0.023 \mathrm{1} / \mathrm{s}$. Rectangular samples underwent five cycles at different maximal applied strains: $30 \%, 50 \%$ or $70 \%$.

\subsection{Tensile loading under optical microscope}

Dog bone samples were mounted on a miniature tensile stage (Linkam TST350) and placed under an optical microscope (Olympus BX60). Five cycles were performed on hydrogels at $0.5 \mathrm{~mm} / \mathrm{s}$ up to $50 \%$ applied strain. At each increase of $2 \%$ of applied strain, individual images of the deformed hydrogel surfaces were taken with a resolution of $0.67 \mu \mathrm{m} /$ pixel.

\subsection{Single edge notch test}

Single edge notch test was performed on rectangular swollen preloaded and non-preloaded neat hydrogels and hydrogel composites with the tensile machine Zwicky. Preloaded samples underwent 5 tensile cycles at constant strain rate of $0.0231 / \mathrm{s}$, which corresponds to a displacement rate of $0.5 \mathrm{~mm} / \mathrm{s}$, up to different applied strains (30\%, 50\% and 70\%) before a $3 \mathrm{~mm}$ crack was cut with a scalpel in the hydrogels (Fig. 1c). The samples were then loaded at $0.5 \mathrm{~mm} / \mathrm{s}$ until rupture. In parallel, the test was recorded with a camera videoXtens (Zwick/Roell) at a $25 \mathrm{fps}$ with a resolution of $0.1 \mathrm{~mm} /$ pixel. The images were used to determine and calculate the variation of the cross-sectional area during the test, providing the true stress.

The fracture strength of notched samples was defined as the maximum stress of the true stress-strain curve, the crack initiation energy density represents the area under the stress-strain curve until the first peak and the fracture energy $\Gamma$ is defined when 


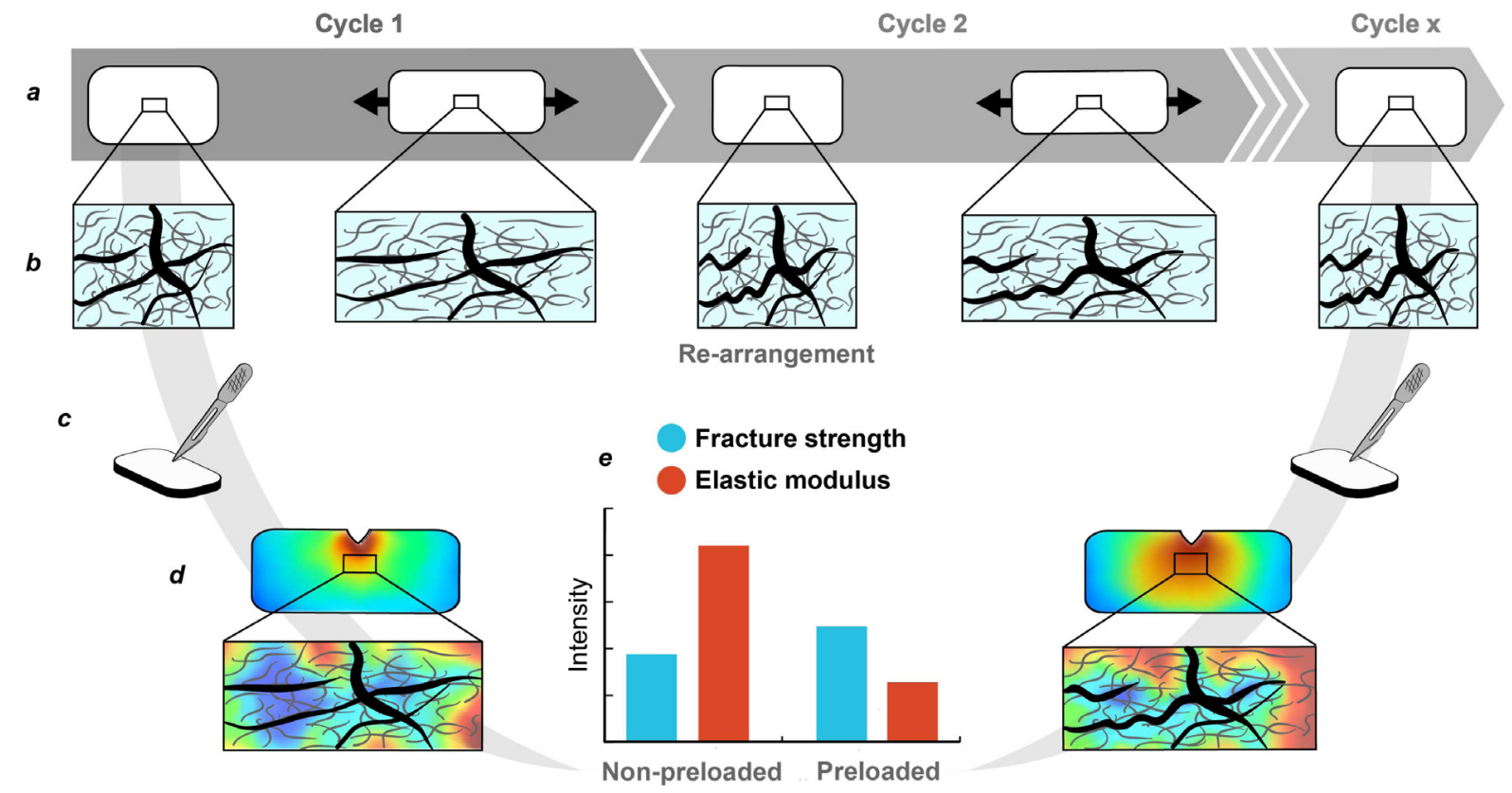

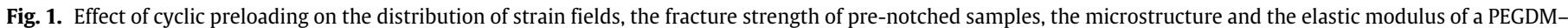

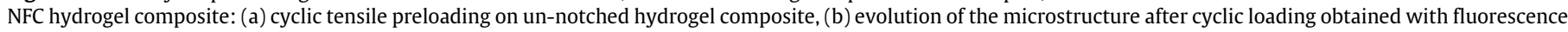

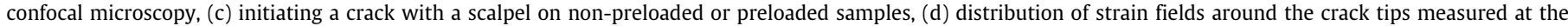

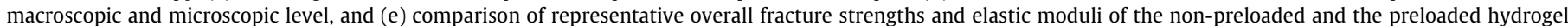
composite.

critical energy release rate $G_{c}$ is reached. $G_{c}$ was characterized with Eq. (2) [14].

$G_{c}=2 \frac{3}{\sqrt{\lambda_{c}}} c W_{s}\left(\lambda_{c}\right)$

where $c$ is the initial crack length and $W_{s}\left(\lambda_{c}\right)$ is the stored energy density of an un-notched sample under the critical stretch $\lambda_{c}$. The critical energy release rate $G_{c}$ is calculated when the stretch $\lambda_{c}$ reaches the first peak of the stress-strain curve of a notched sample

\subsection{Digital image correlation analysis}

Graphit 33 (Kontakt Chemie, Germany) was sprayed on the surface of notched samples to make a fine, randomly and homogeneously distributed speckles pattern. To obtain a finer speckles pattern for DIC at the microscopic level, a drop of Graphit 33 dissolved in isopropanol was deposited on the surface of dog bone samples and wipe a few seconds later with a lens cleaning tissue. Images of the resulting speckles pattern are shown in the Supplementary on Fig. S2. DIC analysis was then performed with the open source software ncorr V1.2 on Matlab R2016b. The references images were taken when the applied load reached $0.04 \mathrm{~N}$.

\subsection{Fluorescence confocal microscopy}

The morphology of the NFC fibers was observed in their native environment with an invert fluorescent confocal microscope (Zeiss LSM 700) equipped with a $20 \times$ lens and a laser of $405 \mathrm{~nm}$. The hydrogel composite was placed for at least $6 \mathrm{~h}$ in $0.2 \mathrm{~g} / \mathrm{l}$ Calcofluor White stain (Sigma-Aldrich, Buchs, $\mathrm{CH}$ ) and 4 vol.\% of $10 \mathrm{wt} \%$ potassium hydroxide in order to dye the NFC fibers.

\section{Results \& discussion}

\subsection{Cyclic tensile loading and mullins effect}

Fig. 2a and b show representative cyclic tensile loadings on dog bone samples of neat hydrogels and hydrogel composites. The applied strain started at $30 \%$ and was progressively increased by $20 \%$ after each 5 th cycle until rupture of the hydrogel.

The elastic tensile modulus was determined by a linear regression between $10 \%$ and $15 \%$ applied strains. It significantly increases from $19 \pm 0.6 \mathrm{kPa}$ to $118 \pm 3.6 \mathrm{kPa}$ when $0.5 \mathrm{wt} \%$ NFC fibers are added to the PEGDM matrix. However, the hydrogel composite becomes softer after the first loading cycle before to remain constant until that the maximum strain previously applied is exceeded, whereas no difference due to cyclic loading is observed for the neat hydrogel.

A previous study [21] made on the same hydrogels revealed a similar behavior in compression and identified it as a Mullins effect. Nevertheless, we observed that, after the first drop of the elastic modulus value, the hydrogel composites withstand 10 million loading cycles at $20 \%$ strain.

However, it is not clear how the hydrogel composites behave during the damage progression. In our previous study, we suggested that cyclic loading on hydrogel composites caused internal damages on the NFC fiber network [21]. Consequently, the fracture properties of a preloaded hydrogel composite would be expected to decrease because a large amount of the mechanical energy input is dissipated during preloading, especially during the first loading cycle as demonstrated by the large hysteresis loop during the first loading-unloading cycle [30,31]. Since no difference was observed on neat hydrogels, the main source for the dissipated energy originated probably from the disintegration of hydrogen bonds of the NFC network (bonds between cellulose-cellulose or between PEGDM-cellulose) [1], from interfacial friction, and from the deformation of NFC fibers.

To better understand the fracture behavior of this material, single notch edge tests were performed on neat hydrogels and hydrogel composites being preloaded or not.

\subsection{Single edge notch test on hydrogels with and without preloading}

Since most softening happens at the first cycle, rectangular hydrogel samples were preloaded 5 times before to create a notch 


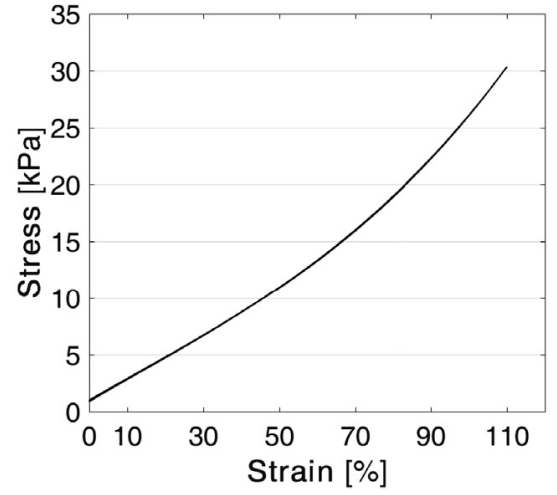

(a) Neat hydrogel

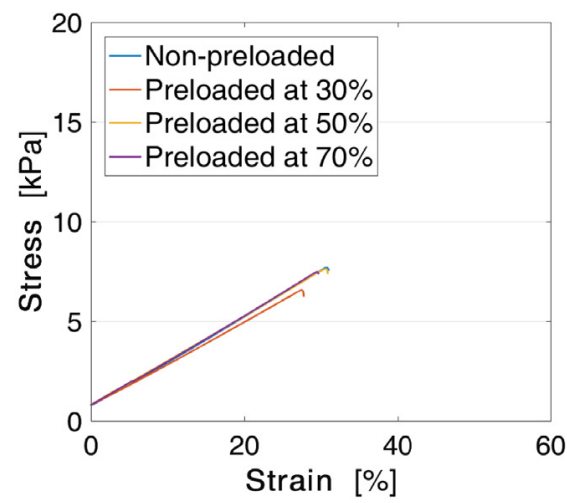

(c) Neat hydrogel

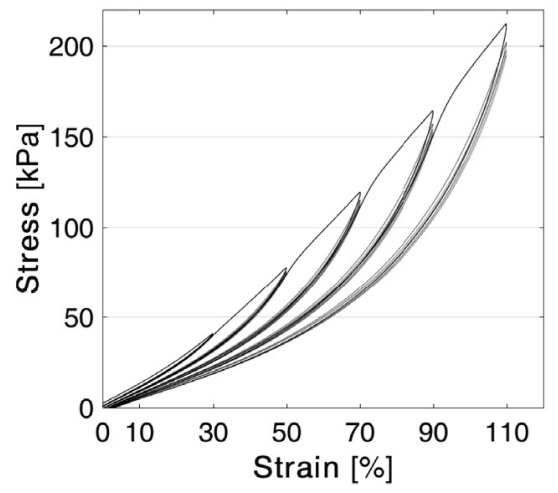

(b) Hydrogel composite

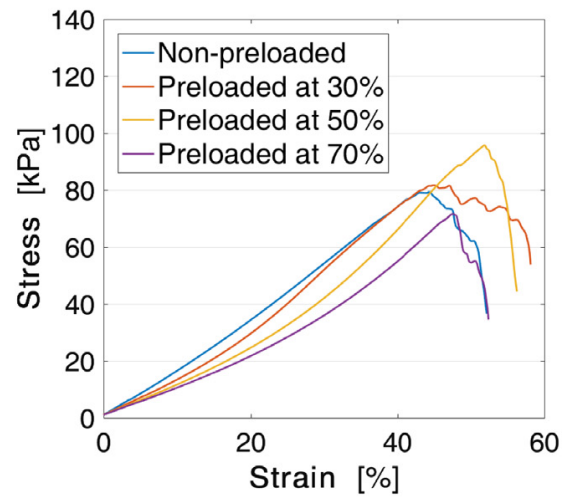

(d) Hydrogel composite

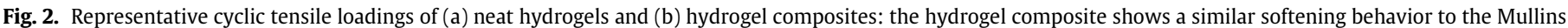

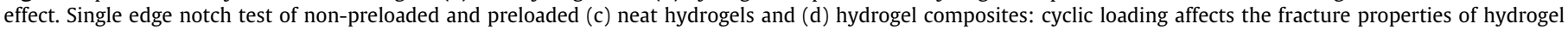
composite, whereas no difference is noticed for the neat hydrogel.

in the material and to perform the single edge notch test. Fig. 2c and $\mathrm{d}$ show the corresponding fracture behavior of non-preloaded or preloaded neat hydrogel and hydrogel composites.

With or without preloading, the fracture strength of notched samples increases more than 10 times with the presence of NFC fibers. The significant improvement is due to (i) the 5 times larger elastic modulus of the hydrogel composites and most probably to (ii) some fibers bridging. Moreover, preloading of hydrogel composites clearly affects their fracture behavior, whereas no difference is detected for neat hydrogels.

Fig. 3 shows the fracture strength of notched samples, the crack initiation energy, and the fracture energy of several non-preloaded and preloaded hydrogel composites at 30\%, 50\% and 70\% applied strain. Note that the fracture strength is conventionally designed for un-notched samples, while the fracture strength reported on Fig. $3 \mathrm{a}$ are measured on notched samples. The creation of a crack reduces therefore significantly their strength and stretchability [34, 35].

The fracture energy $\Gamma$ is commonly used to describe the fracture properties of any materials. It represents the amount of strain energy required to propagate a crack in a pre-notched samples. The fracture energy $\Gamma$ of non-preloaded hydrogels, $223 \pm 10 \mathrm{~J} / \mathrm{m}^{2}$ (Fig. 3c), is in the range of conventional hydrogel composites [36]. Similar to the results obtained on fatigue fracture for double network hydrogels [30,31], the fracture energy decreases progressively with the applied strain during preloading. This behavior was anticipated because of the Mullins effect. Indeed, an increasing softening will reduce the stored energy in an un-notched sample and consequently the available fracture energy (see Eq. (2)). Note that the residual strain after 5 cycles is consequent. Therefore, the stress-strain curves were recalibrated for the calculation of the fracture energy.

However, the fracture strength $\mathrm{S}$ of non-preloaded samples is around $75 \pm 7 \mathrm{kPa}$ and surprisingly increases up to $90 \pm 7 \mathrm{kPa}$ when $30 \%$ strain is applied as preloading. Similarly, the crack initiation energy $\mathrm{W}$, based on notched samples only, increases by $27 \%$ when $50 \%$ strain is applied as preloading. However, after $50 \%$ applied strain, the overall fracture properties start to deteriorate indicating that the hydrogel composite has undergone some irreversible damage.

Several damage phenomena such as fiber bridging, fiber network re-arrangement and decohesion at interfaces may explain the observed variations of fracture strengths, crack initiation and fracture energies. To investigate these phenomena, a multiscale approach was adopted. As a first step, digital image correlation (DIC) analysis was performed in parallel to the single notch edge test to obtain local information on the strain field, in particular around the crack tip $[27,37,38]$.

\subsection{Distribution of strain fields at macroscopic scale}

About 150 images were extracted from the video recorded during the single notch edge test and analyzed with DIC. Fig. 4 shows the Eulerian strain field in the loading direction around the crack tip of hydrogel composites when the crack starts to propagate. Further DIC images at different crack propagation stages can be found in the Supplementary on Fig. S3. The different preloading conditions affect the distribution of the strain fields. This field is qualitatively larger for preloaded hydrogels as shown on Fig. 4. It seems that more regions can deform at higher strains meaning 
a

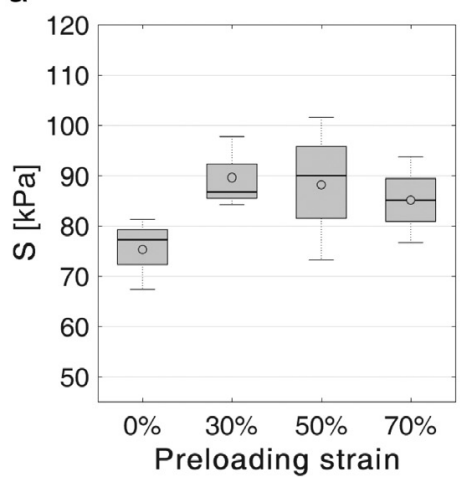

b

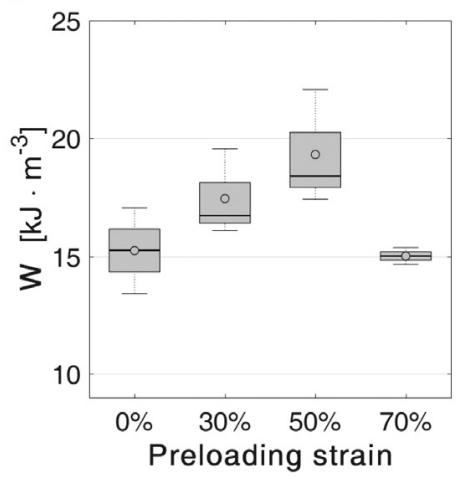

C



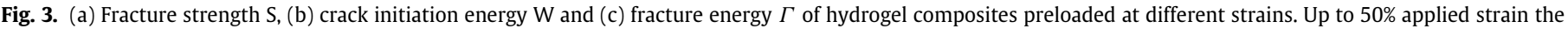
fracture strengths and crack initiation energies increase while the fracture energy decreases.

that more energy can be absorbed and distributed in the material. Since more energy is required for its propagation, crack initiation and propagation are delayed in preloaded materials. In order to explore in more details the complex distribution of strain fields, DIC were performed at the microscopic scale on dog bone hydrogel samples [37].

\subsection{Distribution of the strain fields at microscopic scale}

Considering the size of the NFC fibers (a few micrometers in length [33]), the strain field at the microscopic scale may significantly be influenced in every direction. Therefore, the Lagrangian hydrostatic strain field of un-notched composite and neat hydrogels was observed at the microscopic level (Fig. 5). The dog bone samples were fixed on a miniature tensile machine, placed under an optical microscope and cyclic loaded at 50\% strain along the $\mathrm{x}-$ axis. In parallel, about 30 pictures were extracted at cycle 1 and 5.

Fig. $5 \mathrm{a}$ and $\mathrm{b}$ present the local distribution of strain fields observed in the center of dog bone hydrogel composites at $25 \%$ applied strain in cycle 1 and 5. Further DIC images at different applied strains are shown in the Supplementary on Fig. S4. The strain field of the hydrogel composite is inhomogeneous in comparison to the one of the neat hydrogel. Indeed, the local hydrostatic strain varies from 0.1 to 0.7 . The inhomogeneity is probably related to the NFC fibers distribution. Since NFC fibers are 100'000 time stiffer $(E=1.7$ GPa [33]) than the PEGDM matrix ( $E=20 \pm 0.6 \mathrm{kPa})$, the fibers, in particular the larger ones, that are oriented in the loading direction (x-axis) limit significantly local deformations (blue regions) as suggested on Fig. 1b and mentioned as well by another study [39]. The stress around those large fibers is therefore expected to be high.

Moreover, similar to the above macroscopic results, more regions deform at higher strains in preloaded composites. After loading, compression and tensile regions appear in the hydrogel composite as shown on Fig. 5c. Since no difference is observed in the neat hydrogel (Fig. 5d), the NFC fiber network probably underwent some re-arrangement after the first loading cycle. Furthermore, a previous study showed that the fiber network is probably prestrained after swelling, because (i) the hydrogel composite swells less than the neat hydrogel and (ii) the swelling ratio of the hydrogel composite increases after mechanical tests [21]. Therefore, mechanical loading may also relax some internal stresses in the composite. In order to support the obtained results, the morphology of the studied material was observed with fluorescence confocal microscopy.

\subsection{Direct observation of the NFC morphology}

Fig. 6a and b represent Z-projection and color coded hyperstack images presenting the evolution of the NFC network inside the same hydrogel composite after different preloading conditions: $0 \%, 30 \%, 50 \%$ and $70 \%$ applied strains. The images are taken in the center area of the dog bone sample. The distance between each frame is $0.75 \mu \mathrm{m}$. Each color corresponds to a specific depth and give thus information on the space distribution of the fiber network. The corresponding 3D projections are shown in Movie 1-4 in the Supplementary.

As shown in Fig. 6a and Fig. S5 in the Supplementary, the natural fibers vary in size, morphology and local space distribution. Consequently, their behavior under deformation is expected to differ as well, which explains the observed heterogeneity of the distribution of the strain fields at the microscopic level in Fig. 5a and $\mathrm{b}$.

As shown on Fig. 6a, the hydrogel composite keeps the global three dimensional microstructure of the NFC fibers after being cyclically preloaded (see Movie 5 in the supplementary). However, a small expansion and local modifications of the fiber network appear after preloading. The main damage mechanism is related to the larger fibers oriented in the loading direction that underwent permanent deformations after cyclic loading. The Fig. $6 \mathrm{~b}$ shows their gradual changes in morphology. They probably reached either their failure strength or their elastic limit, which is expressed by the buckling of some NFC fibers. Indeed, after removal of the load, the hydrogel composite returns to its initial position and compresses the freshly elongated fibers that consequently deformes out of their initial planes. The observed damage is favored with the applied strain, inducing a decrease of the elastic modulus and fracture energy. Especially, the overall fracture properties start to deteriorate after $50 \%$ preloading strain because of the to high level of damage.

The NFC fibers are subjected to similar deformations around the crack tip. To observe this, a notch was created in non-preloaded hydrogel. After being dyed with Calcofluor white stain, the notched hydrogel was stretched to propagate the crack a few millimeters and a piece of paper was placed in the crack to keep it slightly open before placing the hydrogel under the confocal microscope. The resulting Z-projections are shown on Fig. 7 and the corresponding $3 \mathrm{D}$ projections in the Movie 6 and 7 in the Supplementary. The morphology of fibers close to the cracks surface suggests that the fibers underwent permanent deformation as well when the crack propagated. Moreover, the cracks circumvented large NFC fibers (Fig. 7A), pulled out (Fig. 7B) or broke (Fig. 7C) some NFC fibers. These observations demonstrate the importance of fibers morphology and bridging phenomena on the toughness of hydrogel 


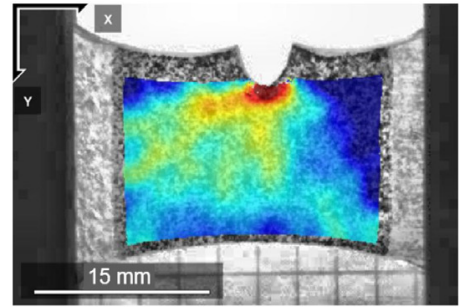

(a) Non-preloaded

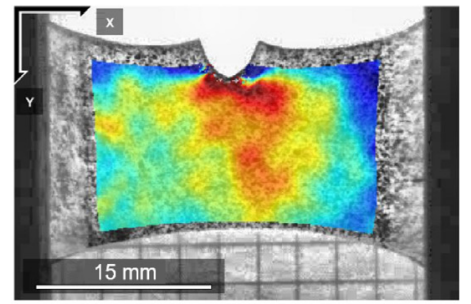

(c) Preloaded at $50 \%$

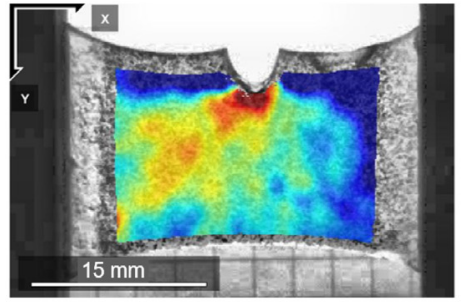

(b) Preloaded at $30 \%$

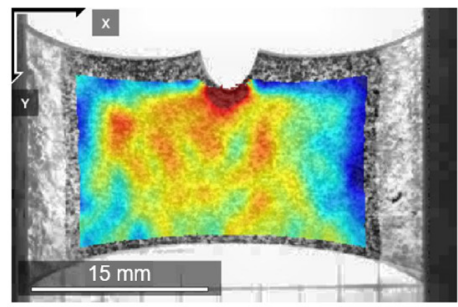

(d) Preloaded at $70 \%$

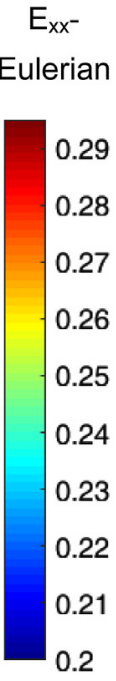

0.2

Fig. 4. Digital image correlation images showing the Eulerian strain along the $x$-axis around the crack tip, which starts to propagate in hydrogel composites preloaded at different strain conditions. The distribution of strain fields becomes larger after preloading.

HS-

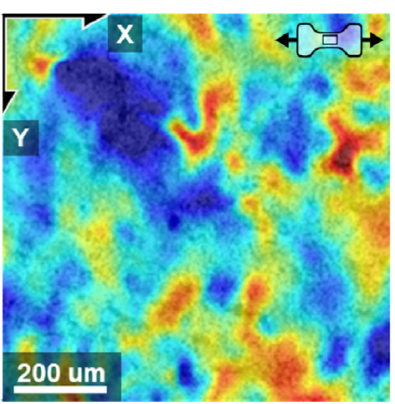

(a) Hydrogel composite - cycle 1 at $25 \%$ applied strain

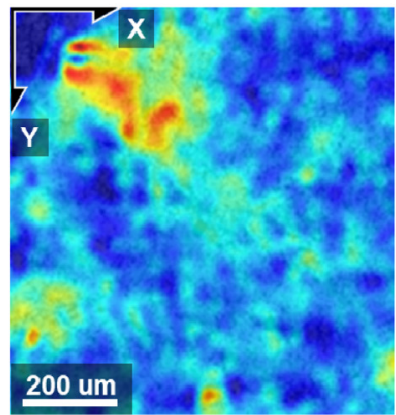

(c) Hydrogel composite - after preloading
Lagrangian

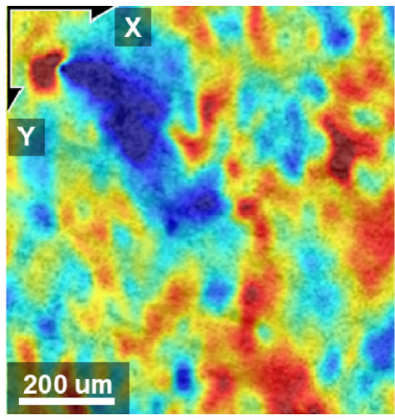

(b) Hydrogel composite - cycle 5

at $25 \%$ applied strain
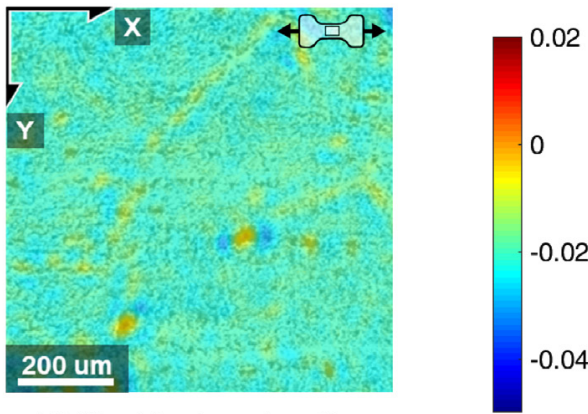

(d) Neat hydrogel - after preloading

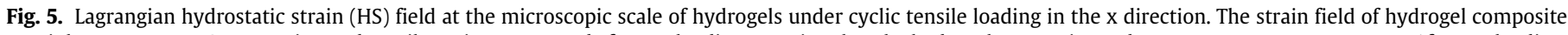

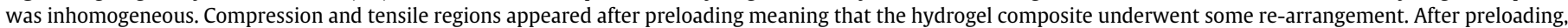
more regions deform at larger strain.

composites. Indeed, as seen in Fig. 7, fibers larger than $20 \mu \mathrm{m}$ in diameters certainly provide a strong bridging effect and thus contribute to the composite toughness.
This study shows that cyclic loading re-arranges locally the fiber network (Fig. 1a and 1b, Fig. 6). During the first loading cycle, some NFC fibers deform plastically or break, which dissipate 
a

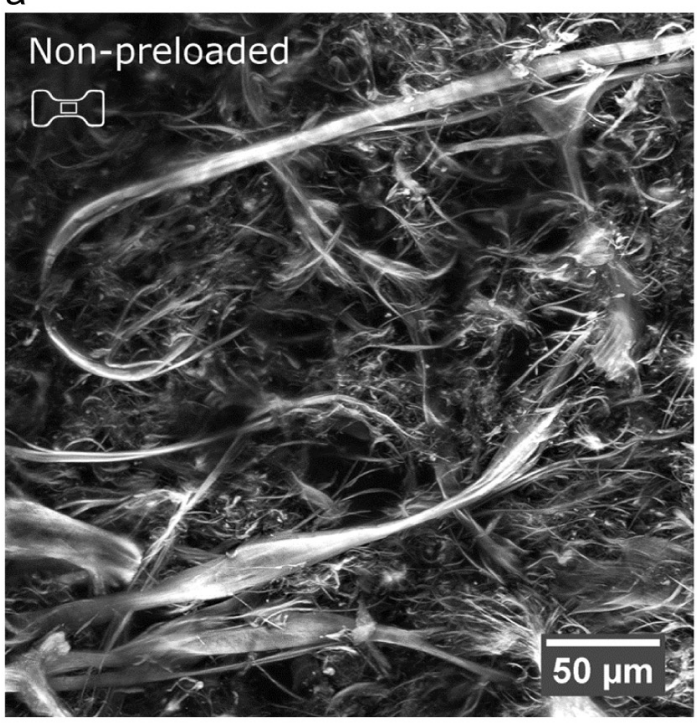

b

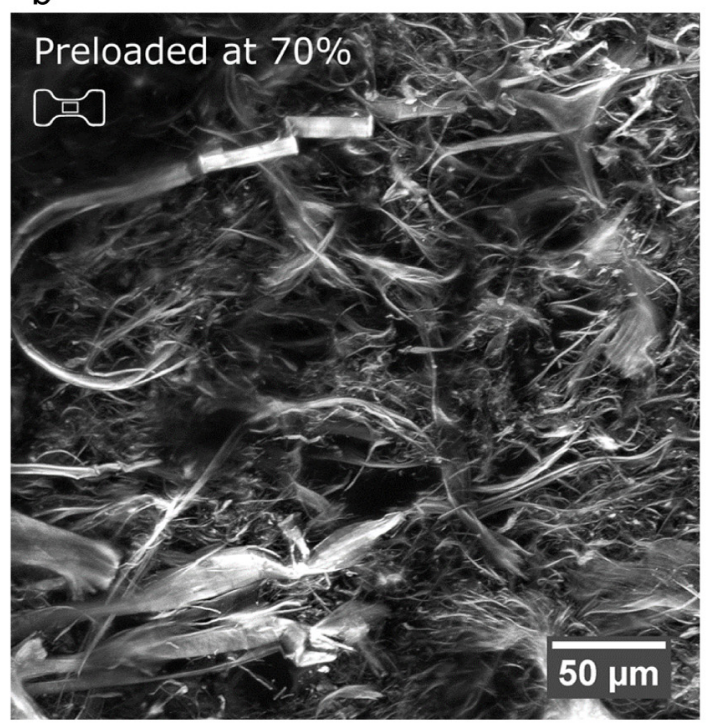

b

\section{Preloading strain}

$\begin{array}{llll}0 \% & 30 \% & 50 \% & 70 \%\end{array}$
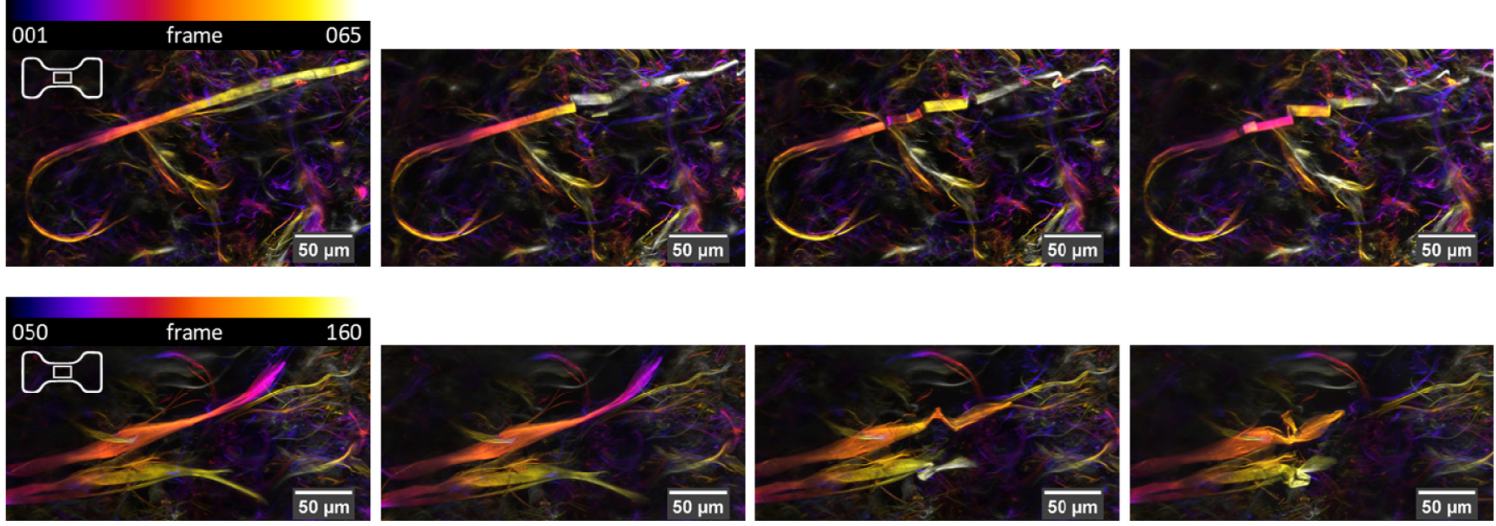

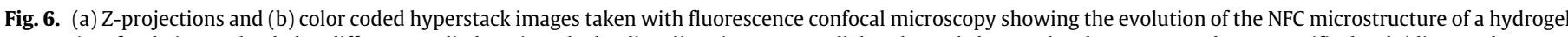

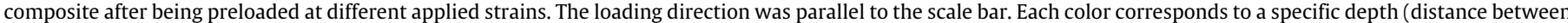

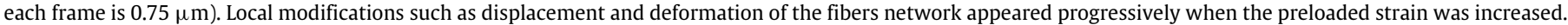

energy as showed with the large hysteresis of the stress-strain curve in Fig. 2b. In the second loading cycle, the hydrogel dissipates much less energy because the re-arranged and/or broken fibers are relaxed. The altered NFC network reduces therefore the available energy for fracture $\Gamma$ in the hydrogel composite and its overall elastic modulus (Figs. 1e, 2b, 3c) but also relaxes some internal stresses. Consequently, the stress concentration around those fibers is diminished and somehow homogenized. As DIC results suggest, the material can deform more easily around these affected regions. During the single edge notch test, the strain is concentrated around the crack tip. In such inhomogeneous strain field conditions, the local energy is related to the strain level. Thus, the energy around the crack tip is higher than elsewhere in the material. DIC results show that the strain distribution around the crack tip becomes larger after cyclic loading. Since more regions in the preloaded material are deformed at higher strains, more energy can be absorbed and distributed in the material strain before the crack starts to propagate (Figs. 1d, 4, 5). Therefore, up to preloading strain level where damage starts to dominate (cf. above $50 \%$ applied strain), preloading increases the total crack initiation energy W (Fig. 3b) and the fracture strength S (Figs. 1e, 3a) of notched samples in contrast to the fracture energy.

\section{Conclusion}

The presence of NFC fibers in PEGDM matrix significantly increases the stiffness and the toughness of the hydrogels. However, similar to the Mullins effect, the hydrogel composite becomes softer after the first loading cycle before to reach a constant mechanical behavior. Preloading the hydrogel composite up to $50 \%$ applied strain surprisingly increases the fracture strength of notched samples and the crack initiation energy by more than $20 \%$, while the corresponding fracture energy decreases.

The results on fibers visualization show that cyclic preloading re-arrange the fiber network, relaxing and reducing residual stresses in the hydrogel composite. The re-arrangement and deformation of the nano-fibrillated cellulose fibers decrease somehow the macroscopic elastic modulus and correspondingly the fracture energy. However, it helps the hydrogel composite to accommodate larger strains before the crack starts to propagate, which 


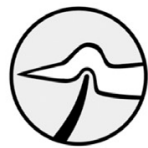

(A) Circumvent



(B) Pull out

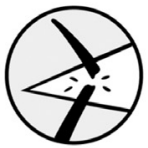

(C) Break

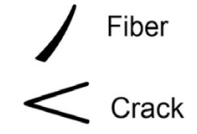

Crack
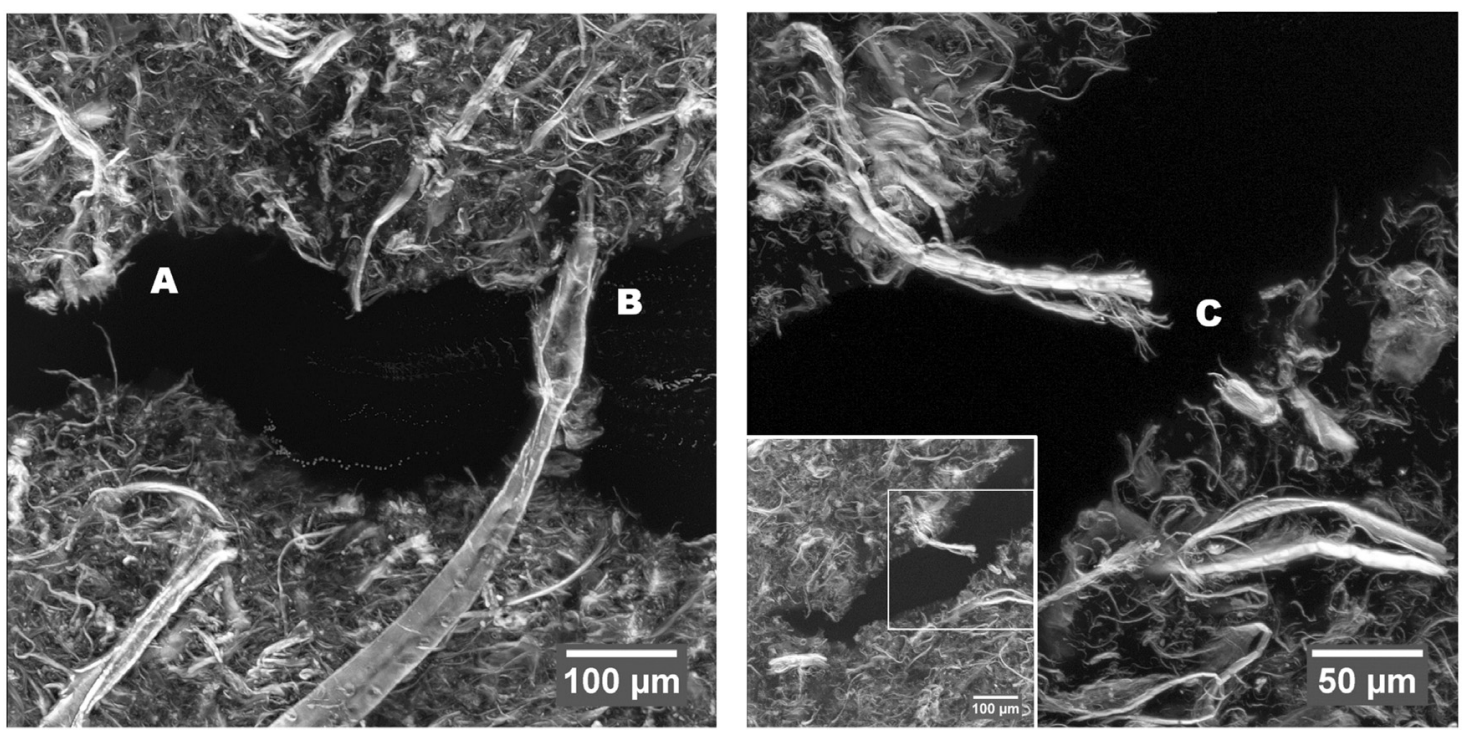

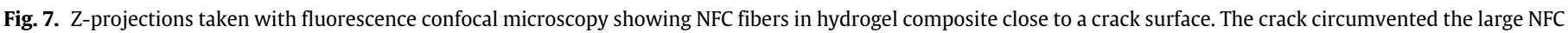
fibers (A), pulled out (B), or broke (C) some NFC fibers, which demonstrates the existence of fiber bridging phenomena in the hydrogel composite.

subsequently improves the fracture strength and the total crack initiation energy of pre-notched sample.

At more than $50 \%$ of applied preloading strain, relaxation and re-arrangement of the fibers network start to be dominated by their damage and failure.

\section{Acknowledgments}

This work was supported by the Swiss National Science Foundation, Switzerland (grant CR 2312-137743). The Applied Wood Materials Laboratory, led by Dr T. Zimmerman at EMPA Dübendorf, Switzerland prepared the NFCs and the BioImaging \& Optics Platform (BIOP) at EPFL provided support for the microscopy.

\section{Appendix A. Supplementary data}

Supplementary material related to this article can be found online at https://doi.org/10.1016/j.eml.2018.09.002.

\section{References}

[1] A.S. Hoffman, Hydrogels for biomedical applications, Adv. Drug Deliv. Rev. 64 (Supplement) (2012) 18-23.

[2] J. Li, D.J. Mooney, Designing hydrogels for controlled drug delivery, Nat. Rev. Mater. 1 (12) (2016) 16071.

[3] K.Y. Lee, D.J. Mooney, Hydrogels for tissue engineering, Chem. Rev. 101 (7) (2001) 1869-1880.

[4] A. Sivashanmugam, R. Arun Kumar, M. Vishnu Priya, S.V. Nair, R. Jayakumar, An overview of injectable polymeric hydrogels for tissue engineering, Eur. Polym. J. 72 (2015) 543-565.

[5] A.M.S. Costa, J.F. Mano, Extremely strong and tough hydrogels as prospective candidates for tissue repair-A review, Eur. Polym. J. 72 (2015) 344-364.

[6] C.W. Peak, J.J. Wilker, G. Schmidt, A review on tough and sticky hydrogels, Colloid Polym. Sci. 291 (9) (2013) 2031-2047.

[7] D.L. Butler, S.A. Goldstein, F. Guilak, Functional tissue engineering: The role of biomechanics, J. Biomech. Eng. 122 (6) (2000) 570-575.

[8] T.M. Maul, D.W. Chew, A. Nieponice, D.A. Vorp, Mechanical stimuli differentially control stem cell behavior: morphology, proliferation, and differentiation, Biomech. Model. Mechanobiol. 10 (6) (2011) 939-953.
[9] G. Altman, et al., Cell differentiation by mechanical stress, FASEB J. 16 (2) (2001) 270-272.

[10] X.X. Shao, D.W. Hutmacher, S.T. Ho, J.C.H. Goh, E.H. Lee, Evaluation of a hybrid scaffold/cell construct in repair of high-load-bearing osteochondral defects in rabbits, Biomaterials 27 (7) (2006) 1071-1080.

[11] O. Jeon, et al., Highly elastic and tough interpenetrating polymer networkstructured hybrid hydrogels for cyclic mechanical loading-enhanced tissue engineering, Chem. Mater. 29 (19) (2017) 8425-8432.

[12] M.A. Haque, T. Kurokawa, J.P. Gong, Super tough double network hydrogels and their application as biomaterials, Polymer 53 (9) (2012) 1805-1822.

[13] P. Lin, S. Ma, X. Wang, F. Zhou, Molecularly engineered dual-crosslinked hydrogel with ultrahigh mechanical strength, toughness, and good selfrecovery, Adv. Mater. 27 (12) (2015) 2054-2059.

[14] K. Mayumi, J. Guo, T. Narita, C.Y. Hui, C. Creton, Fracture of dual crosslink gels with permanent and transient crosslinks, Extreme Mech. Lett. 6 (2016) 52-59.

[15] J.P. Gong, Why are double network hydrogels so tough? Soft Matter 6 (12) (2010) 2583-2590.

[16] E.S. Dragan, Design and applications of interpenetrating polymer network hydrogels. A review, Chem. Eng. J. 243 (2014) 572-590.

[17] S. Utech, A.R. Boccaccini, A review of hydrogel-based composites for biomedical applications: enhancement of hydrogel properties by addition of rigid inorganic fillers, J. Mater. Sci. 51 (1) (2016) 271-310.

[18] F. Zhao, D. Yao, R. Guo, L. Deng, A. Dong, J. Zhang, Composites of polymer hydrogels and nanoparticulate systems for biomedical and pharmaceutical applications, Nanomaterials 5 (4) (2015) 2054-2130.

[19] A. Schmocker, et al., A photopolymerized composite hydrogel and surgical implanting tool for a nucleus pulposus replacement, Biomaterials 88 (2016) $110-119$.

[20] A. Khoushabi, et al., Photo-polymerization, swelling and mechanical properties of cellulose fibre reinforced poly(ethylene glycol) hydrogels, Compos. Sci. Technol. 119 (2015) 93-99.

[21] A. Khoushabi, C.S. Wyss, B. Caglar, D.P. Pioletti, Tailoring swelling to control softening mechanisms during cyclic loading of PEG/cellulose hydrogel composites, Compos.Sci.Technol. 168 (2018) 88-95.

[22] R.E. Webber, C. Creton, H.R. Brown, J.P. Gong, Large strain hysteresis and mullins effect of tough double-network hydrogels, Macromolecules 40 (8) (2007) 2919-2927.

[23] Q. Chen, et al., Improvement of mechanical strength and fatigue resistance of double network hydrogels by ionic coordination interactions, Chem. Mater. 28 (16) (2016) 5710-5720

[24] J.-Y. Sun, et al., Highly stretchable and tough hydrogels, Nature 489 (7414) (2012) 133-136.

[25] Y. Mao, S. Lin, X. Zhao, L. Anand, A large deformation viscoelastic model for double-network hydrogels, J. Mech. Phys. Solids 100 (2017) 103-130. 
[26] J. Diani, B. Fayolle, P. Gilormini, A review on the Mullins effect, Eur. Polym. J. 45 (3) (2009) 601-612.

[27] T. Zhang, S. Lin, H. Yuk, X. Zhao, Predicting fracture energies and crack-tip fields of soft tough materials, Extreme Mech. Lett. 4 (2015) 1-8.

[28] J. Tang, J. Li, J.J. Vlassak, Z. Suo, Fatigue fracture of hydrogels, Extreme Mech. Lett. 10 (2017) 24-31.

[29] E. Zhang, R. Bai, X.P. Morelle, Z. Suo, Fatigue fracture of nearly elastic hydrogels, Soft Matter 14 (18) (2018) 3563-3571.

[30] R. Bai, Q. Yang, J. Tang, X.P. Morelle, J. Vlassak, Z. Suo, Fatigue fracture of tough hydrogels, Extreme Mech. Lett. 15 (2017) 91-96.

[31] W. Zhang, et al., Fatigue of double-network hydrogels, Eng. Fract. Mech. 187 (2018) 74-93.

[32] R. Bai, J. Yang, X.P. Morelle, C. Yang, Z. Suo, Fatigue fracture of self-recovery hydrogels, ACS Macro Lett. 7 (3) (2018) 312-317.

[33] T. Zimmermann, N. Bordeanu, E. Strub, Properties of nanofibrillated cellulose from different raw materials and its reinforcement potential, Carbohydr. Polym. 79 (4) (2010) 1086-1093.
[34] C. Chen, Z. Wang, Z. Suo, Flaw sensitivity of highly stretchable materials, Extreme Mech. Lett. 10 (2017) 50-57.

[35] M. Pharr, J.-Y. Sun, Z. Suo, Rupture of a highly stretchable acrylic dielectric elastomer, J. Appl. Phys. 111 (10) (2012) 104114.

[36] K. Tonsomboon, A.L. Butcher, M.L. Oyen, Strong and tough nanofibrous hydrogel composites based on biomimetic principles, Mater. Sci. Eng. C 72 (2017) $220-227$.

[37] S. Hong, et al., 3D printing of highly stretchable and tough hydrogels into complex, cellularized structures, Adv. Mater. 27 (27) (2015) 4035-4040.

[38] M.A. Gonzalez, et al., Strong, tough, stretchable, and self-adhesive hydrogels from intrinsically unstructured proteins, Adv. Mater. 29 (10)(2017) 1604743

[39] M. Mehdikhani, M. Aravand, B. Sabuncuoglu, M.G. Callens, S.V. Lomov, L. Gorbatikh, Full-field strain measurements at the micro-scale in fiber-reinforced composites using digital image correlation, Compos. Struct. 140 (2016) 192201. 